\title{
KEY TO THE ABBREVIATIONS USED IN THE BIBLIOGRAPHY
}

Amer. Anthrop.

American Anthropologist

Ann. Rpt. Bur. Amer. Ethnol.

Annual Report of the Bureau of

American Ethnology

Australian Mus. Mag.

Australian Museum Magazine

Bernice P. Bishop Mus.

Bernice P. Bishop Museum Press

Bernice P. Bishop Mus. Bull. Bernice P. Bishop Museum Bulletin

Bernice P. Bishop Mus. Mem. Bernice P. Bishop Museum Memoirs

Bernice P. Bishop Mus. Occas. Papers

Bernice P. Bishop Museum

Occasional Papers

Californian

The Californian

Cram's Mag.

Cram's Magazine

Friend.

The Friend

Hawaiian Hist. Soc.

Ann. Rpt.

Hawaiian Historical Society

Annual Report

Hawaiian Hist. Soc.

Hawaiian Historical Society

Hawaiian Hist. Soc. Papers.

Hawaiian Historical Society Papers

Hawaiian Jour. Hist.

Hawaiian Journal of History

Hon. Adv.

Honolulu Advertiser

Hon. Almanac.

Honolulu Almanac

Hon. Mercury.

Honolulu Mercury

Hon. Star-Bull.

Honolulu Star-Bulletin

Hon. Star-Bull. \& Adv.

Honolulu Star-Bulletin \& Advertiser

Hutchings' Calif. Mag.

Hutchings' California Magazine

Internatl. Quart.

International Quarterly

Islander.

The Islander

Jour. Amer. Folklore

Journal of American Folklore

Jour. Polynesian Soc.

Journal of the Polynesian Society

Kamehameha

The Kamehameha Schools Press

Lit. Rev.

Literary Review

Maile Quart.

Maile Quarterly

Mid-Pac. Mag

Mid-Pacific Magazine

New Zealand Inst. Trans. and Proc.

New Zealand Institute, Transactions and

Proceedings

Pac. Com. Adv.

Pacific Commercial Advertiser

Pac. Monthly

Pacific Monthly

Pan-Pac.

. Pan-Pacific

Par. Pac.

Paradise of the Pacific

Polynesian. The Polynesian

Thrum's Ann.

Thrum's Annual for. . .

T. K. K. Topics

Toyo Kisen Kaisha Topics

Tongg.

. Tongg Publishing $\mathrm{Co}_{\mathrm{C}}$

Tuttle.

Charles E. Tuttle Co.

UHP

University of Hawaii Press

UPH

The University Press of Hawaii 